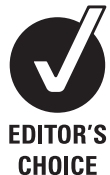

Department of Medicine, Division of Infectious Diseases, Johns Hopkins University School of Medicine, Baltimore, Maryland, USA

\section{Correspondence to}

Dr Khalil G Ghanem, JHUBMC ID Division, 4940 Eastern Ave B3N, Baltimore, MD 21224 USA; kghanem@jhmi.edu

Accepted 18 July 2010 Published Online First 5 October 2010

\title{
Treatment of syphilis in HIV-infected subjects: a systematic review of the literature
}

\author{
Leah J Blank, Anne M Rompalo, Emily J Erbelding, Jonathan M Zenilman, \\ Khalil G Ghanem
}

\begin{abstract}
Background The optimal antimicrobial regimen to treat syphilis in HIV-infected subjects remains controversial. Objective To systematically assess the literature for studies evaluating syphilis treatment regimens in this population.
\end{abstract}

Methods Two reviewers independently assessed studies published between 1980 and June 2008 in electronic databases, trial registries and bibliographies (with no language restrictions) for content and quality. Studies that included 10 or more people, with documented HIV status, type and duration of syphilis treatment and at least 6 months of follow-up were included. The primary outcome was syphilis serological or clinical failure stratified by syphilis stage.

Results Of 1380 unique citations, 23 studies (22 published papers and 1 conference abstract) were included in the systematic review. Owing to the significant heterogeneity among studies, pooled summary statistics could not be generated. The range of probabilities for serological failure with 2.4 million units (MU) of intramuscular benzathine penicillin $\mathrm{G}$ (BPG) was $6.9 \%(95 \% \mathrm{Cl} 2.6 \%$ to $14.4 \%)$ to $22.4 \%(11.7 \%$ to $36.6 \%)$; that of $7.2 \mathrm{MU}$ of $\mathrm{BPG}$ in late latent syphilis was $19.4 \%(11.9 \%$ to $28.9 \%)$ to $31.1 \%(22.3 \%$ to $40.9 \%)$ and failure estimates with 18-24 MU of aqueous penicillin for the treatment of neurosyphilis were $27.3 \%(6.0 \%$ to $61.0 \%)$ to $27.8 \%(14.2 \%$ to $45.2 \%)$.

Conclusions The optimal antimicrobial regimen to treat syphilis in HIV-infected subjects is unknown; guideline recommendations in this population are based on little objective data.

\section{INTRODUCTION}

Since its introduction in the 1940s, penicillin has remained the preferred drug for treating syphilis. Data from randomised trials comparing the efficacy of the different penicillin-based syphilis treatment regimens are limited. ${ }^{1}$ Currently, the Centers for Disease Control and Prevention (CDC) recommend the use of long-acting intramuscular benzathine penicillin G (BPG) for the treatment of early and late latent syphilis and high-dose intravenous aqueous crystalline penicillin $G$ for the treatment of neurosyphilis. ${ }^{2}$ Even though Treponema pallidum is isolated in the cerebrospinal fluid of up to $40 \%$ of people with early syphilis, ${ }^{3}{ }^{4}$ and BPG does not achieve treponemicidal levels in the cerebrospinal fluid, ${ }^{5}$ there were very few reports of late manifestations of syphilis in people treated with penicillin in the pre-HIV era. ${ }^{6}$

The optimal treatment regimen for syphilis in HIV-infected subjects has been controversial since case reports emerged in the late 1980s documenting failures to CDC-recommended treatment regimens. ${ }^{7}$ Several studies have suggested an increased risk of syphilis treatment failure when standard regimens are used while others have not. ${ }^{8}$ The current CDC recommendations suggest using the same treatment regimens as those used in HIVuninfected subjects with more aggressive posttreatment serological follow-up in those who are co-infected. ${ }^{2}$ Our goal was to systematically assess the literature for studies reporting syphilis serological and clinical treatment responses in HIV infected subjects stratified by the stage of syphilis infection.

\section{METHODS}

\section{Data sources and searches}

We searched Medline, SCOPUS, ISI, POPLINE, Excerpta Medica and Cochrane Central Register of Controlled Trials databases from 1980 to June 2008 (or earliest date available within the time range). We used the following search terms: syphilis AND (treatment $\mathrm{OR}$ therapy $\mathrm{OR}$ management) AND ('HIV infection' OR 'HIV infections' OR 'HIV persons' OR 'HIV patient'). Reference lists of the retrieved articles were also manually searched for other potentially relevant papers. We also reviewed conference abstracts (International Society for Sexually Transmitted Diseases Research, CDC STD Prevention Conferences, Infectious Diseases Society of America meetings, Interscience Conference on Antimicrobial Agents and Chemotherapy, Conference on Retroviruses and Opportunistic Infections). We assessed both English and non-English language articles for eligibility.

\section{Study selection}

We evaluated articles for eligibility using a twostage procedure. In the first stage, all identified abstracts were reviewed. In the second stage, we reviewed the entire text of articles that met the inclusion criteria and articles whose eligibility was uncertain based on review of the abstract. Where information was missing, we attempted to contact either the primary or senior authors on the study to request additional information or clarification. All articles were independently assessed by two reviewers.

The inclusion criteria for studies were (1) syphilis diagnosis based on serological criteria or darkfield microscopy; patients treated based on syndromic management without serological or microscopic confirmation were excluded; (2) 10 or more people with at least one patient documented HIV positive; 
(3) the HIV status of the patient was known at or near ( \pm 365 days) the time of syphilis diagnosis; (4) the type and duration of antimicrobial therapy received was documented; (5) clinical or serological outcome was reported with at least 6 months of follow-up after syphilis treatment. The primary outcome was syphilis serological or clinical failure at or greater than 6 (early-stage syphilis) to 12 (late latent syphilis or neurosyphilis) months after treatment. We used the definition for serological non-response as a fourfold increase in non-treponemal titres 30 or more days after appropriate treatment or lack of a fourfold decline in non-treponemal titres 6-12 months after treatment for early syphilis or 12-24 months for late syphilis (instances where follow-up was less than the times specified above are highlighted in the tables). ${ }^{2}$ For neurosyphilis, the definitions of failure included lack of a fourfold or greater non-treponemal serological response at or after 12 months after treatment and/or the lack of improvement in cerebrospinal fluid abnormalities (white blood cell count, Venereal Disease Research Laboratory test or protein), or clinical signs suggestive of neurosyphilis after 12 months.

\section{Data abstraction, quality assessment and analyses}

Both reviewers (LJB and KGG) independently extracted data from all primary studies that fulfilled the inclusion criteria; disagreement was resolved by consensus. The following information was abstracted: study design, syphilis staging, type and duration of syphilis treatment, post-treatment follow-up time and adherence, stage-specific serological and clinical outcomes and assessment of methods to account for re-infection as a cause of syphilis treatment failure. We summarised outcomes for these studies by tabulating the number of failures by the total number of subjects who completed follow-up at the specified time. If studies assessed outcomes at more than one follow-up time, all times beyond the 6-month time point were recorded and the time point with the least amount of loss to follow-up is reported in the tables. We scored the methodological quality of the studies by evaluating study design, allocation concealment, blinding, loss to follow-up and source of funding, as applicable. ${ }^{9}$

Our initial goal was to perform a meta-analysis. However, owing to the significant heterogeneity in treatment used, adherence and follow-up, we were unable to generate meaningful pooled summary statistics. Instead we summarised the data of each individual study and tabulated the results. We calculated probability of serological failure for each study stratified by syphilis stage and treatment regimen used. We provide $95 \%$ confidence limits for point estimates to describe the degree of uncertainty surrounding each value.

\section{RESULTS}

Of 1380 unique citations, we excluded 1342 on initial screening; 38 studies were fully reviewed: 37 were published articles and one was a conference abstract (figure 1). Fifteen articles did not meet one or more entry criteria and were excluded. The remaining 23 studies, 22 published papers ${ }^{10-32}$ and one conference abstract, ${ }^{33}$ which has since been published, ${ }^{34}$ were included in the systematic review after both reviewers agreed on their validity assessments.

Studies that met inclusion criteria included: six retrospective case series, seven prospective case series, two case-control studies, two retrospective cohort studies, three prospective cohort studies and four randomised trials (table 1). Two of the trials were deemed good quality based on Jadad scores of $5 .^{19} 27$ Two randomised trials compared efficacy of azithromycin with

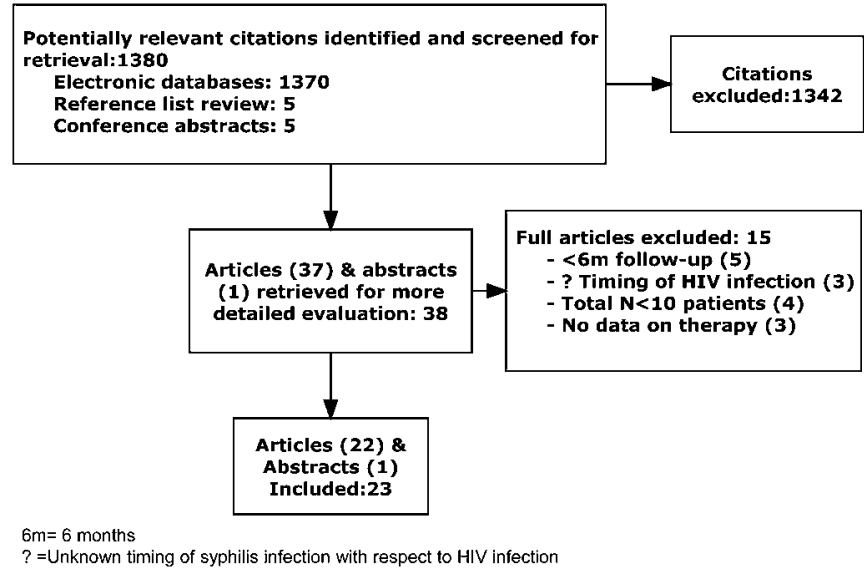

Figure 1 Study flow diagram.

other regimens and included HIV-infected subjects among the recruited participants, ${ }^{21}{ }^{27}$ one small randomised open-label study in HIV-infected subjects compared penicillin with ceftriaxone in late latent and neurosyphilis, ${ }^{23}$ and one randomised open label trial compared BPG with enhanced treatment with additional amoxicillin and probenecid among subjects with early syphilis. ${ }^{19}$ The latter trial included subjects with HIV. The remaining studies were descriptive.

Most of the studies reported data on multiple treatment regimens for various stages of syphilis resulting in very small numbers within each category. Some studies only reported failure rates for aggregate data inhibiting our ability to distinguish the efficacy of each individual treatment regimen. Studies that fulfilled entry criteria and reported failures for each regimen with a sample size of $>10 \mathrm{HIV}$-positive persons for that regimen are reported (table 2). The range of probabilities for serological failure with 2.4 million units (MU) of intramuscular BPG was $6.9 \%$ (95\% CI $2.6 \%$ to $14.4 \%$ ) to $22.4 \%$ (11.7\% to $36.6 \%$ ); that of 7.2 MU of BPG in late latent syphilis was $19.4 \%(11.9 \%$ to $28.9 \%)$ to $31.1 \%(22.3 \%$ to $40.9 \%)$ and failure estimates with 18-24 MU of aqueous penicillin for the treatment of neurosyphilis were $27.3 \%(6.0 \%$ to $61.0 \%)$ to $27.8 \%$ (14.2\% to $45.2 \%)$. Only one of the randomised trials ${ }^{27}$ was included in table 2 because the data from the other three trials were reported in aggregate or because the number of HIV-infected subjects, when stratified by stage and drug regimen, was fewer than 10 .

\section{DISCUSSION}

The current treatment guidelines in both the USA ${ }^{2}$ and the $\mathrm{UK}^{35}$ recommend BPG as first-line treatment for early and late latent syphilis in both HIV-infected and uninfected subjects. Assuming lack of heterogeneity, were we to pool data from the 23 studies included in this systematic review, serological outcomes of people treated with BPG (2.4 MU intramuscularly for early syphilis and 7.2 MU intramuscularly for late latent syphilis) could be assessed in a total of 197 people with early and 245 people with late latent syphilis. There are even fewer data available on the use of doxycycline (100 mg orally twice a day) to treat early syphilis in HIV-infected subjects: 20 total cases. For neurosyphilis, outcomes on 68 people treated with intravenous aqueous penicillin (18-24 MU intravenously for 10-14 days) and 19 people treated with ceftriaxone $(2 \mathrm{~g}$ intramuscularly or intravenously daily for 10-14 days) could be assessed. Our study entry criteria were not overly restrictive. In fact, we chose criteria that would allow for the least restrictive clinically meaningful 


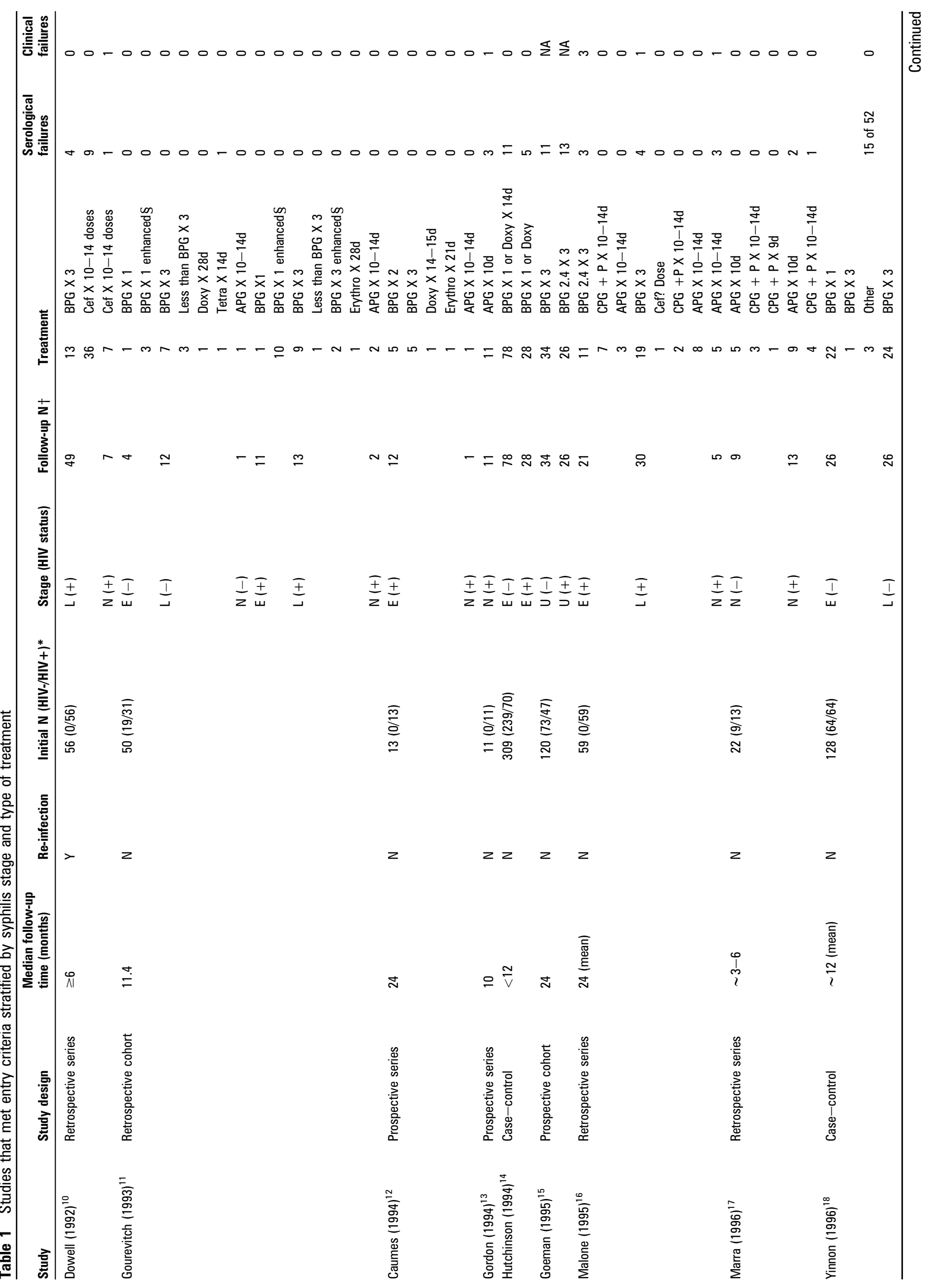




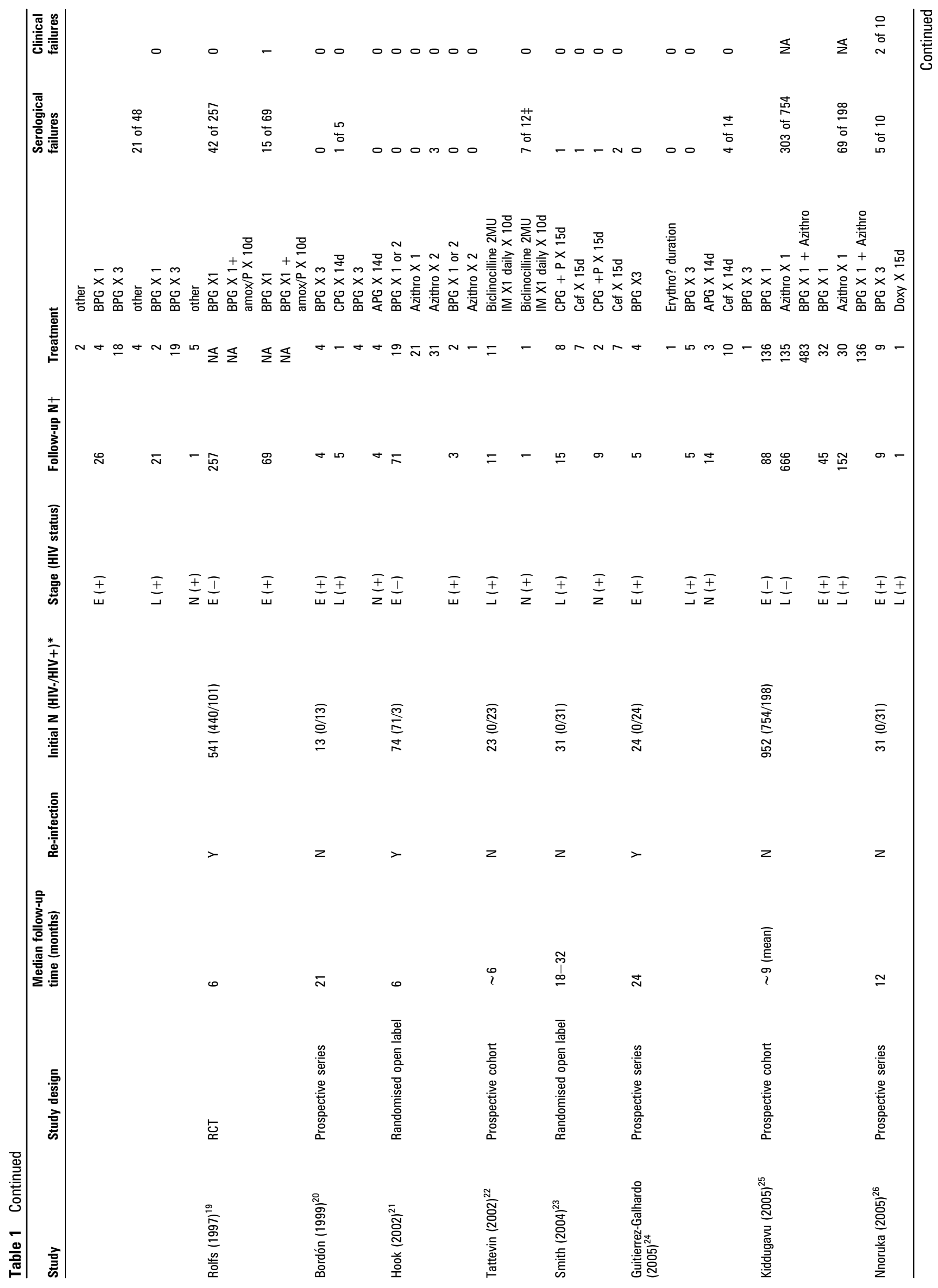




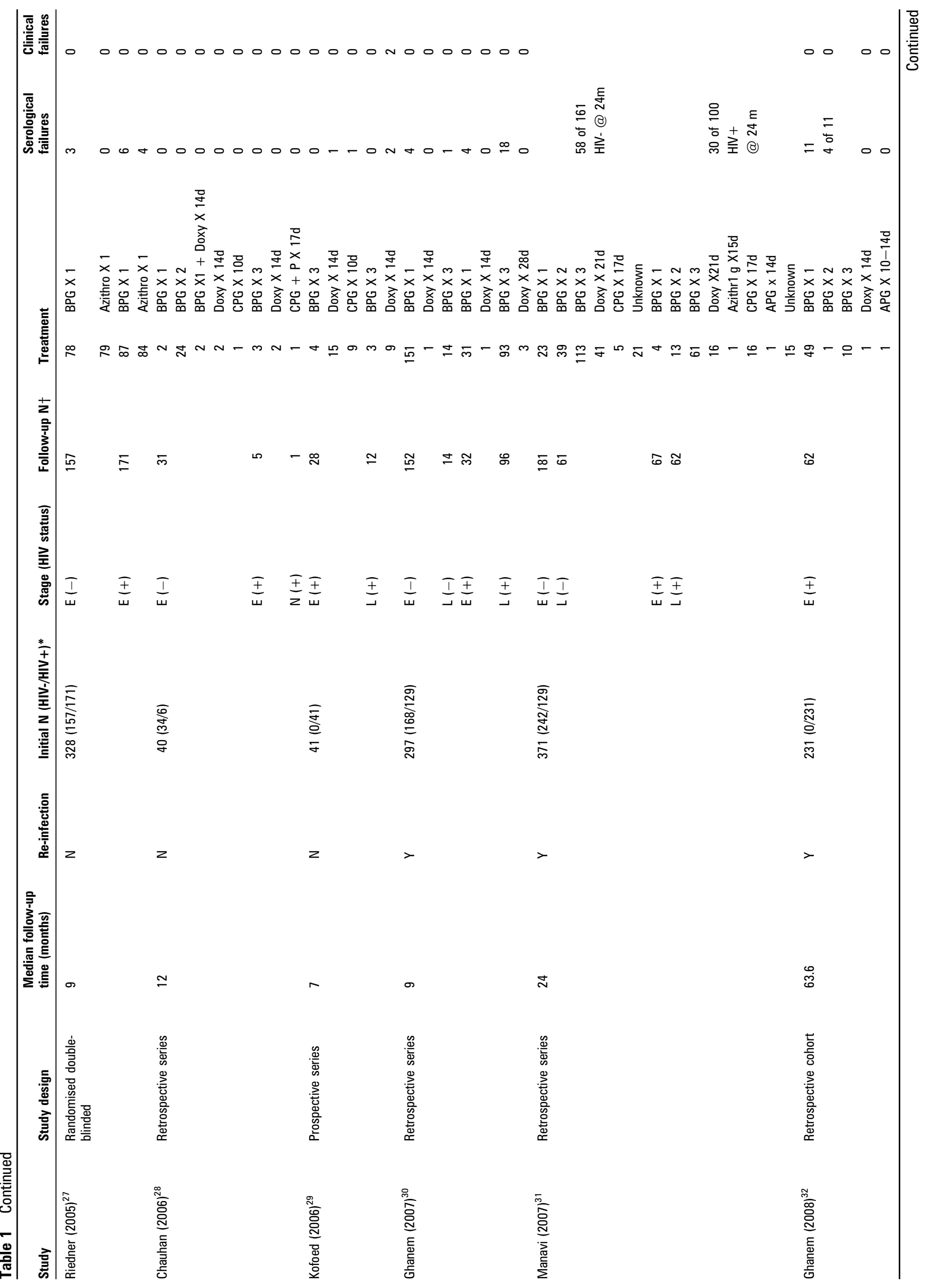




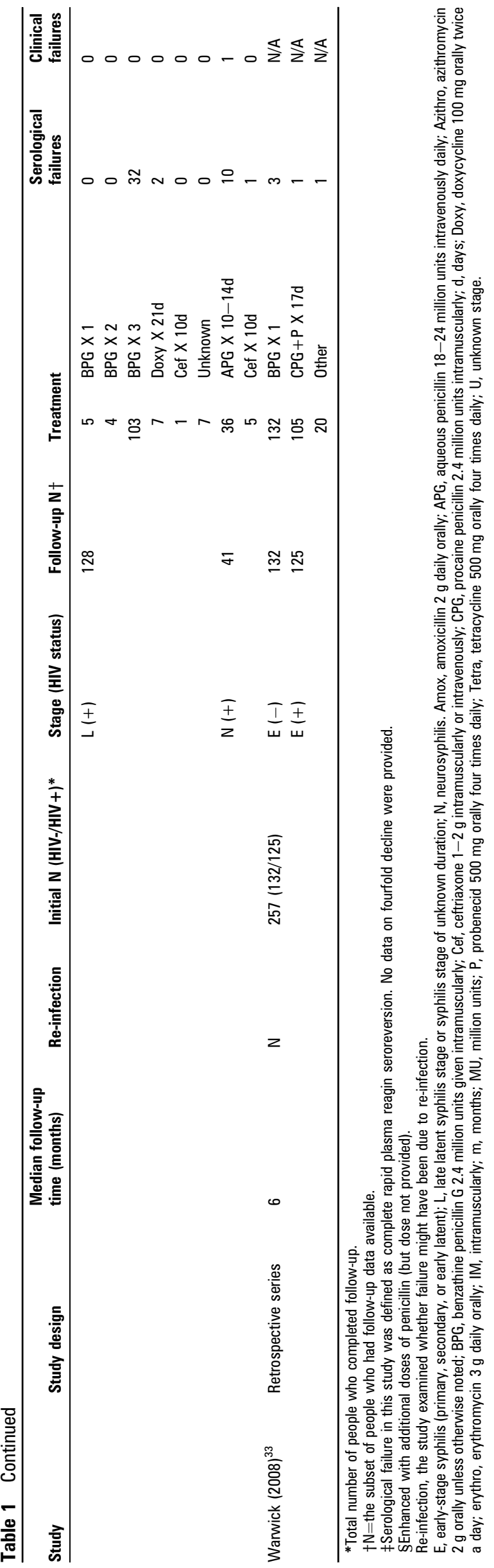

interpretation of data. Even higher-quality randomised trials did not evaluate the efficacy of syphilis treatment in HIV-positive subjects as a primary outcome. The most commonly cited paper to justify the use of similar treatment regimens in HIV-infected and uninfected subjects is a randomised controlled trial comparing BPG with enhanced treatment $\mathrm{BPG}+$ amoxicillin and probenecid) in people with early syphilis. ${ }^{19} \mathrm{~A}$ total of 541 people were enrolled, but only 101 were HIV-infected and, of those, only 69 completed follow-up at 6 months. Although no difference in response rate between the treatment regimens used was noted, HIV-infected subjects with primary syphilis were found to have higher serological failure rates than HIV-uninfected subjects. Of note, the augmented treatment consisting of amoxicillin and probenecid chosen for the study had never been shown to be adequate treatment for neurosyphilis.

Of the studies summarised in table 2, only a few had HIVuninfected controls. Of those, the probability of failure among HIV-uninfected subjects was lower than for HIV-infected subjects, although most comparisons were not statistically significant owing to small numbers. Among HIV-uninfected subjects, the historical estimate of treatment failure with 2.4 MU of BPG is $5 \%,{ }^{1}$ an estimate similar to those seen among HIV-uninfected subjects in our studies. The estimates among HIV-infected subjects (table 2) range from $1 \%$ to $22 \%$-the high estimate was from a cohort study that could not account for the possibility of reinfection. ${ }^{32}$ The two treatment regimens in table 2 that approach the historical efficacy of $5 \%$ are single-dose azithromycin and procaine penicillin plus probenecid. In the past several years, mutations associated with azithromycin resistance have been detected in people infected with syphilis. ${ }^{36-38}$ Thus, the routine use of azithromycin to treat early syphilis is not recommended. The $1 \%$ failure rate seen with the procaine penicillin regimen is, by far, the best estimate reported..$^{34}$ Indeed, among all the regimens for early syphilis, this regimen is the only one approved for the treatment of neurosyphilis. One hypothesis is that the enhanced efficacy of this regimen is due to its potential to treat occult underlying neurosyphilis. Previously, UK treatment guidelines recommended this as the preferred regimen to treat HIV-infected patients with early syphilis; however, the recent updated UK guidelines favour BPG instead.

The estimates for treatment failures among HIV-infected subjects with late latent syphilis and neurosyphilis are much higher than historical estimates from HIV-uninfected subjects, which range from $3 \%$ to $10 \% .{ }^{1}$ One possibility is that the recommended penicillin regimens are less effective in HIVinfected subjects. There are, however, plausible alternate hypotheses. In late latent syphilis, slower serological response times among those with a previous history of treated syphilis ${ }^{39}$ and among HIV-infected subjects have been reported. In most studies, the median follow-up times were less than the 24 months suggested by the $\mathrm{CDC}{ }^{2}$ This might have inflated the rates of serological failures. When assessing treatment responses for neurosyphilis, HIV-infected subjects, especially those with advanced HIV, can have baseline cerebrospinal fluid abnormalities unrelated to syphilis. ${ }^{40}$ This may have an impact on the case definition and failure rates reported for neurosyphilis. These issues highlight a major factor that has hindered progress in understanding the natural history of syphilis: the main clinical measure of syphilis disease activity, serological titres, are not specific for treponemes and may not reflect the underlying microbiology. Interpretation of serological titres is even more difficult in the setting of HIV where B-cell dysfunction may account for some of the observed variability. ${ }^{41}$ 
Table 2 Probability of treatment failure for various therapeutic regimens stratified by syphilis stage and HIV status (if data on HIV-uninfected subjects were reported). Studies that fulfilled entry criteria and reported failures for each regimen with a sample size of $>10$ people for that regimen are included

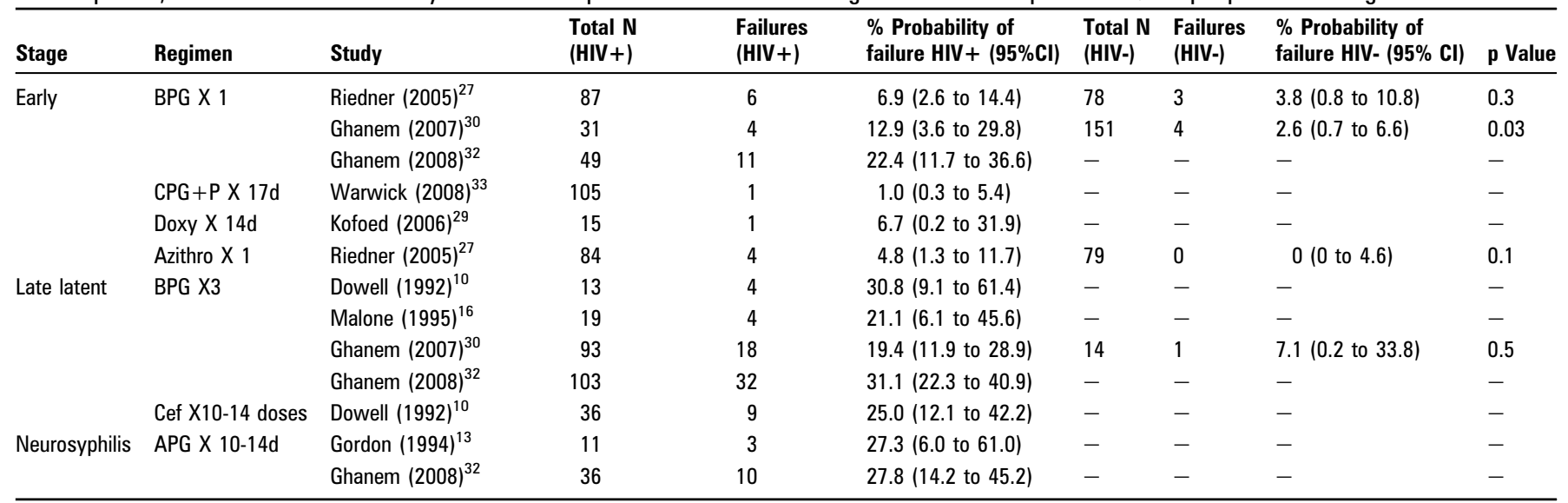

APG, aqueous penicillin 18-24 million units intravenously daily; Azithro, azithromycin $2 \mathrm{~g}$; BPG, benzathine penicillin $\mathrm{G} 2.4$ million units given intramuscularly; Cef, ceftriaxone 1-2 g intramuscularly or intravenously; CPG, procaine penicillin 2.4 million units intramuscularly; d, days; Doxy, doxycycline 100 mg orally twice a day; m, months; $\mathrm{N}$, number; $\mathrm{P}$, probenecid 500 mg orally four times daily; -, no data available for HIV-uninfected subjects.

Given the limitations of the data, one approach has been to advocate conservative approaches to managing co-infected people: cerebrospinal fluid examination in all HIV-infected subjects with syphilis or, at least, the use of treatment regimens that are effective at treating underlying occult neurosyphilis irrespective of syphilis stage. ${ }^{42} 43$ The arguments against broad implementation of these strategies include limited resources and lack of studies demonstrating improved long-term outcomes compared with standard management approaches. ${ }^{44}$ Currently, the main recommendation among co-infected people is for aggressive serological and clinical follow-up after stage-appropriate treatment - a logical recommendation, but one not based on objective evidence of efficacy. ${ }^{2}$ Even this goal, however, is not easily achieved. Among co-infected people attending Baltimore City STD clinics, 64\% did not have documented evidence of any follow-up serologies after syphilis treatment. ${ }^{30}$

This study has several limitations: we were unable to pool results owing to significant heterogeneity among the studies; any such attempts would have yielded uninterpretable data. A significant number of studies did not report outcomes for individual treatment regimens. We attempted to contact all first and senior authors listed to try to obtain clarifications. Although most responded to the enquiries, many reported that they no longer had easy access to the primary data given that some of these studies were 10-15 years old. Our study entry criteria were broad. For example, the CDC treatment guidelines suggest that low rapid plasma reagin titres (eg, titres $<1: 32$ ) in late latent syphilis may not decline fourfold, even after appropriate treatment. Few of the studies we reviewed examined this topic. That is because even the CDC treatment guidelines are fairly opaque about the significance of this finding, suggesting that 'in these circumstances, the need for treatment or repeated cerebrospinal fluid examinations is unclear'. Finally, there is no simple objective way to distinguish between treatment failure and re-infection and most studies made no attempt to do so (table 1). As such, observed differences in treatment responses between HIV-infected and uninfected subjects may be the result of behavioural confounding.

A recent survey of infectious diseases practitioners highlights the pervading uncertainty among clinicians who manage HIVinfected subjects with syphilis. ${ }^{45}$ of 375 clinicians who were queried about the treatment of secondary syphilis in

\section{Key messages}

Studies assessing the treatment of syphilis among HIV-infected subjects are heterogeneous.

- Current guidelines for the treatment of syphilis among HIV-infected subjects are based on limited objective data.

- The optimal antimicrobial regimen to treat syphilis in HIV-infected subjects is unknown.

HIV-infected patient, $64 \%$ used a non-CDC recommended regimen consisting of three doses of $2.4 \mathrm{MU}$ of intramuscular BPG. In an accompanying editorial titled "When guidelines don't guide the physician", the author suggested that the inconsistency seen among clinicians is the result of limited evidence to support clear management approaches. ${ }^{46}$ Our study highlights the significant heterogeneity that exists among treatment studies and the difficulty in trying to make use of these data to guide management decisions. Rates of syphilis continue to increase and HIV-infected subjects are disproportionately affected. ${ }^{47}$ The optimal treatment regimen for syphilis in HIV-infected patients is unknown and, consequently, any guideline recommendation in this population is ultimately based on limited objective data.

Acknowledgements We would like to thank Drs Edward (Ned) Hook III, Michael Augenbraun, Zoe Warwick and Gillian Dean for providing us with additional information on their studies.

Funding Funding from the National Institutes of Health (K23HD047395) was provided to $\mathrm{KGG}$.

\section{Competing interests None}

Contributors LJB: data collection, data abstraction, drafting of the manuscript. AMR/EJE/JMZ: data assessment, drafting of manuscript. KGG: data collection, data abstraction, data analysis and drafting of the manuscript.

Provenance and peer review Not commissioned; externally peer reviewed.

\section{REFERENCES}

1. Idsoe 0, Guthe T, Willcox RR. Penicillin in the treatment of syphilis. The experience of three decades. Bull World Health Organ 1972;47:1-68. 
2. Workowski KA, Berman SM; Centers for Disease Control and Prevention. Sexually transmitted diseases treatment guidelines, 2006. MMWR Recomm Rep 2006;55 (RR-11):1-94

3. Lukehart SA, Hook EW 3rd, Baker-Zander SA, et al. Invasion of the central nervous system by Treponema pallidum: implications for diagnosis and treatment. Ann Intern Med 1988;109:855-62.

4. Marra CM, Maxwell CL, Smith SL, et al. Cerebrospinal fluid abnormalities in patients with syphilis: association with clinical and laboratory features. J Infect Dis 2004:189:369-76.

5. Dunlop EM, Al-Egaily SS, Houang ET. Penicillin levels in blood and CSF achieved by treatment of syphilis. JAMA 1979;241:2538-40.

6. Perdrup A, Jorgensen BB, Pedersen NS. The profile of neurosyphilis in Denmark: A clinical and serological study of all patients in Denmark with neurosyphilis disclosed in the years 1971-1979 incl. by Wassermann reaction (CWRM) in the cerebrospinal fluid. Acta Derm Venereol 1981:96:1-14.

7. Berry CD, Hooton TM, Collier AC, et al. Neurologic relapse after benzathine penicillin therapy for secondary syphilis in a patient with HIV infection. $N$ Engl J Med 1987;316:1587-9.

8. Zetola NM, Klausner JD. Syphilis and HIV infection: an update. Clin Infect Dis 2007:44:1222-8.

9. Jadad AR, Moore RA, Carroll D, et al. Assessing the quality of reports of randomized clinical trials: is blinding necessary? Control Clin Trials 1996;17:1-12.

10. Dowell ME, Ross PG, Musher DM, et al. Response of latent syphilis or neurosyphilis to ceftriaxone therapy in persons infected with human immunodeficiency virus. $A m \mathrm{~J}$ Med 1992;93:481-8.

11. Gourevitch MN, Selwyn PA, Davenny K, et al. Effects of HIV infection on the serologic manifestations and response to treatment of syphilis in intravenous drug users. Ann Intern Med 1993;118:350-5.

12. Caumes E, Minta D, Niel G, et al. Syphilis precoce et infection par le VIH: A propos de 13 cas. Med Mal Infect 1994;24:785-91.

13. Gordon SM, Eaton ME, George R, et al. The response of symptomatic neurosyphilis to high-dose intravenous penicillin $\mathrm{G}$ in patients with human immunodeficiency virus infection. N Engl J Med 1994;331:1469-73.

14. Hutchinson CM, Hook EW 3rd, Shepherd M, et al. Altered clinical presentation of early syphilis in patients with human immunodeficiency virus infection. Ann Intern Med 1994;121:94-100.

15. Goeman J, Kivuvu M, Nzila N, et al. Similar serological response to conventional therapy for syphilis among HIV-positive and HIV-negative women. Genitourin Med 1995;71:275-9.

16. Malone JL, Wallace MR, Hendrick BB, et al. Syphilis and neurosyphilis in a human immunodeficiency virus type-1 seropositive population: Evidence for frequent serologic relapse after therapy. Am J Med 1995;99:55-63.

17. Marra CM, Longstreth WT Jr, Maxwell CL, et al. Resolution of serum and cerebrospinal fluid abnormalities after treatment of neurosyphilis. Influence of concomitant human immunodeficiency virus infection. Sex Transm Dis 1996;23:184-9

18. Yinnon AM, Coury-Doniger P, Polito R, et al. Serologic response to treatment of syphilis in patients with HIV infection. Arch Intern Med 1996;156:321-5.

19. Rolfs RT, Joesoef MR, Hendershot EF, et al. A randomized trial of enhanced therapy for early syphilis in patients with and without human immunodeficiency virus infection. The Syphilis and HIV Study Group. N Engl J Med 1997;337:307-14.

20. Bordon J, Martinez-Vazquez C, de la Fuente-Aguado J, et al. Response to standard syphilis treatment in patients infected with the human immunodeficiency virus. Eur $\mathrm{J}$ Clin Microbiol Infect Dis 1999;18:729-32.

21. Hook EW 3rd, Martin DH, Stephens J, et al. A randomized, comparative pilot study of azithromycin versus benzathine penicillin $\mathrm{G}$ for treatment of early syphilis. Sex Transm Dis 2002;29:486-90.

22. Tattevin $\mathbf{P}$, Renault $\mathrm{P}$, Joly $\mathrm{V}$, et al. Treatment of latent syphilis in HIV-infected patients with $10 \mathrm{~d}$ of benzylpenicillin $\mathrm{G}$ benethamine: A prospective study in Maputo, Mozambique. Scand J Infect Dis 2002;34:257-61.

23. Smith NH, Musher DM, Huang DB, et al. Response of HIV-infected patients with asymptomatic syphilis to intensive intramuscular therapy with ceftriaxone or procaine penicillin. Int J STD AIDS 2004;15:328-32.
24. Gutierrez-Galhardo MC, do Valle GF, Sa FC, et al. Clinical characteristics and evolution of syphilis in 24 HIV + individuals in Rio de Janeiro, Brazil. Rev Inst Med Trop Sao Paulo 2005;47:153-7.

25. Kiddugavu MG, Kiwanuka N, Wawer MJ, et al. Effectiveness of syphilis treatment using azithromycin and/or benzathine penicillin in Rakai, Uganda. Sex Transm Dis 2005:32:1-6.

26. Nnoruka EN, Ezeoke AC. Evaluation of syphilis in patients with HIV infection in Nigeria. Trop Med Int Health 2005;10:58-64.

27. Riedner G, Rusizoka M, Todd J, et al. Single-dose azithromycin versus penicillin $\mathrm{G}$ benzathine for the treatment of early syphilis. N Engl J Med 2005;353 1236-44.

28. Chauhan M, Serisha B, Sankar KN, et al. Audit of the use of benzathine penicillin, post-treatment syphilis serology and partner notification of patients with early infectious syphilis. Int J STD AIDS 2006;17:200-2.

29. Kofoed K, Gerstoft J, Mathiesen LR, et al. Syphilis and human immunodeficiency virus (HIV)-1 coinfection: influence on CD4 T-cell count, HIV-1 viral load and treatment response. Sex Transm Dis 2006;33:143-8.

30. Ghanem KG, Erbelding EJ, Wiener ZS, et al. Serological response to syphilis treatment in HIV-positive and HIV-negative patients attending sexually transmitted diseases clinics. Sex Transm Infect 2007:83:97-101.

31. Manavi K, McMillan A. The outcome of treatment of early latent syphilis and syphilis with undetermined duration in HIV-infected and HIV-uninfected patients. Int J STD AIDS 2007:18:814-18.

32. Ghanem KG, Moore RD, Rompalo AM, et al. Antiretroviral therapy is associated with reduced serologic failure rates for syphilis among HIV-infected patients. Clin Infect Dis 2008:47:258-65

33. Should early syphilis be treated differently in HIV positive and HIV negative individuals? Brooklyn, NY. BASHH ASTDA 3rd Joint Conference, Brooklyn, NY, 2008 Abstract E-08.

34. Warwick Z, Dean G, Fisher M. Should syphilis be treated differently in HIV-positive and HIV-negative individuals? Treatment outcomes at a university hospital, Brighton, UK. Int J STD AIDS 2009;20:229-30

35. Kingston $\mathbf{M}$, French $\mathrm{P}$, Goh $\mathrm{B}$, et al. UK National guidelines on the management of syphilis 2008. Int J STD AIDS 2008:19:729-40.

36. Stamm LV, Bergen HL. A point mutation associated with bacterial macrolide resistance is present in both 23S rRNA genes of an erythromycin-resistant Treponema pallidum clinical isolate. Antimicrob Agents Chemother 2000;44: 806-7.

37. Lukehart SA, Godornes C, Molini BJ, et al. Macrolide resistance in Treponema pallidum in the United States and Ireland. N Engl J Med 2004;351:154-8.

38. Matejkova P, Flasarova M, Zakoucka $\mathrm{H}$, et al. Macrolide treatment failure in a case of secondary syphilis: a novel A2059G mutation in the 23S rRNA gene of Treponema pallidum subsp. pallidum. J Med Microbiol 2009;58:832-6.

39. Romanowski B, Sutherland R, Fick GH, et al. Serologic response to treatment of infectious syphilis. Ann Intern Med 1991:114:1005-9.

40. Marra CM, Maxwell CL, Collier AC, et al. Interpreting cerebrospinal fluid pleocytosis in HIV in the era of potent antiretroviral therapy. BMC Infect Dis 2007:7:37.

41. De Milito A. B lymphocyte dysfunctions in HIV infection. Curr HIV Res 2004;2:11-21

42. Marra CM. Déjà Vu all over again: When to perform a lumbar puncture in HIVinfected patients with syphilis. Sex Transm Dis 2007;34:145-6.

43. Ghanem KG, Moore RD, Rompalo AM, et al. Lumbar puncture in HIV-infected patients with syphilis and no neurologic symptoms. Clin Infect Dis 2009:48 816-21

44. Stoner BP. Current controversies in the management of adult syphilis. Clin Infect Dis 2007:44(Suppl 3):S130-46.

45. Dowell D, Polgreen PM, Beekmann SE, et al. Dilemmas in the management of syphilis: a survey of infectious diseases experts. Clin Infect Dis 2009:49:1526-9.

46. Gross PA. When guidelines don't guide the physician. Clin Infect Dis 2009:49:1530-1.

47. Centers for Disease Control and Prevention. Sexually transmitted disease surveillance, 2008. Atlanta, GA: Department of Health and Human Services, 2009. 